\title{
A comparison of Imaging Fourier Transform with Grating Spectrometry for Tridimensional Spectroscopy
}

\author{
Peter L. Smith \\ Center for Astrophysics, 60 Garden Street, Cambridge, MA 02138, USA
}

Anne P. Thorne

Blackett Laboratory, Imperial College, London SW7 2BZ, UK

W. H. Parkinson

Center for Astrophysics, 60 Garden Street, Cambridge, MA 02138, USA

\begin{abstract}
We compare the relative merits of four different types of instrument for tridimensional spectrometry at visible and ultraviolet wavelengths: the echelle and long-slit spectrographs and two types of Fourier transform spectrometer (conventional scanning and fixed tilt spatially heterodyned). Each of these instruments requires different spatial and spectral scanning modes. It is shown that in certain limiting conditions all four are equally efficient, but that they differ in other important characteristics.
\end{abstract}

\section{Introduction}

Two-dimensional array detectors can be used with a number of different spectrometric instruments for 3-D spectroscopy, i.e., the recording of spectral information for a number of points in an extended spatial field. Choice of the best instrument must be guided by the application. The comparison presented in this paper is directed towards broad band spectral coverage at wavelengths from the near infrared into the vacuum ultraviolet (VUV), thus covering space-based as well as ground-based astrophysics. Four instruments are considered: the stigmatic long-slit spectrograph (LSS), the echelle spectrograph (ES), and two types of Fourier transform (FT) spectrometer. The Fabry-Perot interferometer is not included because of its small free spectral range and its very low finesse at VUV wavelengths.

Implicit in our comparison is the assumption that scanning in at least one of the three dimensions is required to record all the required spatial and spectral information with a two-dimensional detector. The property of primary interest is taken to be the efficiency, as measured by the signal-to-noise ratio (SNR) achievable for the same spectral and spatial resolution and the same total observation time on the assumption that the noise is due to photon noise only. We have assumed, for the baseline comparison, that both fields are full, i.e., that the target is an extended object emitting a quasi-continuous spectrum. 
Some different requirements and performance criteria are considered in the last section.

The LSS and the ES are both familiar instruments that will not be described here. The LSS yields spatial information perpendicular to the dispersion direction in its focal plane (the $y$-axis) and the spectrum is dispersed along the $x$-axis. The instantaneous spatial coverage in the $x$-direction is set by the slit width, and 1-D "pushbroom" scanning is required to obtain information in the $x$-direction.

The ES presents instantaneous wide-band spectral coverage in a 2-D format on the detector, but the entrance slit must be limited in height as well as in width in order to separate the spatial and spectral information. A 2-D raster is required to record the spatial information.

An imaging FT spectrometer (IFTS) for the infrared region has been described at this Colloquium by Maillard (1994). The spatial field is imaged directly onto the detector and, as the interferometer is scanned, an interferogram is recorded for each spatial point and subsequently transformed to give its spectrum. The use of IFTS for space-based observations from the visible to the VUV has been discussed by Thorne (1992), and our recent work has established the viability of FTS as a VUV technique in practice. The length of the scan determines the spectral resolution, and the number of independent points in the interferogram is equal to that in the final spectrum.

The spatial heterodyne spectrometer (SHS), a version of the FT spectrometer, has been described by Butcher et al. (1989) and by Harlander \& Roesler (1990). The interferogram is imaged on the detector, but the localisation of the fringes is such that there is no spatial imaging in the $x$-direction: every point in the entrance aperture contributes to every point in the interferogram. A cylindrical lens or mirror can be used to image the $y$-dimension of the entrance aperture on the detector. The optimum strategy for obtaining spatial information in the $x$-direction is rotation of the instrument followed by tomographic reconstruction of the image. (Bétrémieux et al. (1993) describe use of a grating spectrometer in a tomographic manner; we will not consider this option here.)

\section{Assumptions and scanning requirements}

As a basis for our comparisons, we assumed that all the instruments have the same input focal ratio and optical efficiency, that the field of view is matched to the detector size, and that there is no "dead" time between scans. Nyquist sampling requires two pixels per resolution element, so we shall consider pixel pairs throughout the comparison. In this unit, the dimensions of the detector are $p \times p$, the required spatial resolution is $x \times y$, and the required spectral resolution is $w$. The spectrum is assumed to be quasi-continuous, with a bandwidth of $N$ independent spectral points where $N \geq p$.

The spectral scanning requirements are as follows: the IFTS records $N$ interferogram points in one scan; the SHS records $p$ interferogram points simultaneously, i.e., $p / N$ of the total; the LSS records $p / w$ spectral points simultaneously, which is $p /(w N)$ of the total; and the ES records the entire spectrum simultaneously, provided $w h \leq p^{2} / N$, where $h$ is the slit height. In the LSS and ES, the effective signal is proportional to the photon flux falling on each pixel pair, or set of binned pixel pairs, multiplied by the allowed integration time. 
For a constant total observation time, the latter is the inverse of the number of scans required. The square root of the effective signal gives the relative SNR in the spectrum. In the two interferometers, the same process yields the SNR in the interferogram, and the SNR in a quasi-continuous spectrum is obtained from this by dividing by the square root of the number of interferogram points.

The spatial scanning requirements for three of the instruments are straightforward. For the SHS we assume the "tomographic" mode, as it can be shown that the alternative of restricting the entrance aperture is less efficient by a factor of $\sqrt{x / p}$.

\section{Relative signal-to-noise ratios}

A summary of the efficiencies of the SHS, LSS and ES relative to that of the IFTS, as measured by the SNRs achievable for observations yielding the same spectral and spatial resolution for the same total time, is presented in Table 1. The efficiencies depend on the relative sizes of the spectral and spatial resolution elements, and Table 1 takes the most favourable case. If the widths of the

Table 1. Relative SNR for the conditions $w<x$ and $h<y$

\begin{tabular}{rcccc}
\hline & IFTS & SHS & LSS & Echelle \\
\hline number of binned spatial pixels & $x y$ & $p y$ & $w y$ & $w h$ \\
ratio to IFTS & 1 & $p / x$ & $w / x$ & $\leq p^{2} / N x y$ \\
photon flux per observing step & $N w$ & $w$ & $w$ & $w$ \\
ratio to IFTS & 1 & $1 / N$ & $1 / N$ & $1 / N$ \\
rasters in $x$-direction & 1 & $p / x$ & $p / x$ & $p / x$ \\
rasters in $y$-direction & 1 & 1 & 1 & $p / y$ \\
rasters in $\lambda$-direction & $N$ & $N / p$ & $N w / p$ & 1 \\
product $[x, y, \lambda]$ rasters & $N$ & $N / x$ & $N w / x$ & $p^{2} / x y$ \\
relative signal $\left(\times N^{2}\right)$ & $N$ & $p$ & 1 & $\leq 1$ \\
SNR in spectrum & 1 & 1 & 1 & $\leq 1$ \\
\hline \hline
\end{tabular}

entrance slit in the LSS and ES are dictated by the spatial rather than the spectral resolution requirement, i.e., if the condition $w<x$ is reversed, the $w$ in lines 1 and 3 of Table 1 must be replaced by $x$, leading to a factor $x^{2} / w^{2}$ in the relative signal and $x / w$ in the SNR. If the slit height in the ES is restricted, (i.e., $y<h$ ), to satisfy spatial rather than spectral resolution requirements, $h$ must be replace by $y$ in the first line of Table 1 , leading to a factor of $y / h$ in the relative signal and of $\sqrt{y / h}$ in the SNR. These changes are simply the result of the unnecessary higher resolution and shorter slit forced by the spatial requirements. Their effects are summarized in Table 2. The SHS in aperturelimited, rather than tomographic, mode is also included in this table. 
Table 2. Relative SNR for different conditions

\begin{tabular}{ccccc}
\hline & IFTS & SHS & LSS & Echelle \\
\hline$w<x, h<y$ & 1 & 1 & 1 & $\leq 1$ \\
$w>x, h<y$ & 1 & 1 & $x / w$ & $x / w$ \\
$w>x, h>y$ & 1 & 1 & $x / w$ & $x / w \sqrt{y / h}$ \\
SHS in aperture-limited mode & 1 & $\sqrt{x / p}$ & $x / w$ & $x / w \sqrt{y / h}$ \\
\hline \hline
\end{tabular}

\section{Other important features}

The implication from Table 1, viz., that the four instruments considered are equally efficient, is true only in the special case that all spectral and spatial elements contain equally useful information. If the spatial field is not full, the ES gains most from reduction in spatial scanning requirements; the LSS and SHS may also gain; the IFTS gains nothing. If the spectral field is not full, e.g., an emission-line spectrum is being observed, the FT and the SHS gain by the inverse of the "filling factor", i.e. by the ratio of filled to empty spectral elements. The LSS gains only if there are large gaps that need not be scanned; and the ES gains nothing. Note that $x$ and $w$ are both set by the entrance slit width in the LSS and ES, whereas in the two FT instruments they are decoupled. The IFTS has three other potential advantages that could, for certain applications, be very significant. Its resolution is entirely flexible (any desired value can be chosen simply by setting the scan length appropriately); the interferometer scan is potentially much faster than the part-spatial, part-spectral scans of the other instruments (an important feature for observations of dynamic processes); and, whereas grating instruments require wavelength standards throughout the spectral region of interest, the FT yields an intrinsically linear wavenumber scale, which can be made absolute with, in principle, only one reference line.

Acknowledgments. The authors thank a number of colleagues, especially J. W. Brault, R. C. M. Learner and M. C. E. Huber, for helpful discussions. This work was supported in part by the Harvard-Smithsonian Center for Astrophysics and NASA Grant NAGW-1596.

\section{References}

Bétrémieux, Y., Cotton, D. M., Cook, T., \& Chakrabarti, S. 1993, Optical Eng., 32,3133

Butcher, H., Douglas, N., Fransden, S. \& Maaswinkel, F. 1989, Opt. Soc. Am. Technical Digest Ser., 6, 9

Harlander, J. \& Roesler, F. L. 1990, Proc. SPIE 1235, 622

Maillard, J.-P., 1994, these proceedings

Thorne, A. P., Proc. ESA Workshop on Solar Physics and Astrophysics at Interferometric Resolution, Paris, 1992 